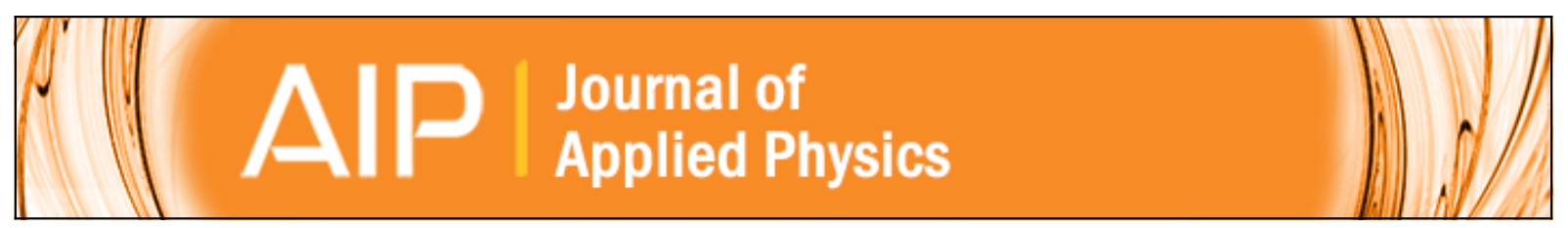

\title{
Near-flat self-biased magnetoelectric response in geometry gradient composite
}

Yuan Zhou and Shashank Priya

Citation: Journal of Applied Physics 115, 104107 (2014); doi: 10.1063/1.4868340

View online: http://dx.doi.org/10.1063/1.4868340

View Table of Contents: http://scitation.aip.org/content/aip/journal/jap/115/10?ver=pdfcov

Published by the AIP Publishing

\section{Articles you may be interested in}

Self-biased large magnetoelectric coupling in co-sintered $\mathrm{Bi} 0.5 \mathrm{Na} 0.5 \mathrm{TiO} 3$ based piezoelectric and $\mathrm{CoFe} 2 \mathrm{O} 4$ based magnetostrictive bilayered composite

J. Appl. Phys. 116, 244101 (2014); 10.1063/1.4904758

Giant self-biased magnetoelectric coupling in co-fired textured layered composites

Appl. Phys. Lett. 102, 052907 (2013); 10.1063/1.4791685

Tunable self-biased magnetoelectric response in homogenous laminates

Appl. Phys. Lett. 101, 232905 (2012); 10.1063/1.4769365

Strong magnetoelectric charge coupling in stress-biased multilayer-piezoelectric magnetostrictive composites J. Appl. Phys. 101, 124102 (2007); 10.1063/1.2748712

Small dc magnetic field response of magnetoelectric laminate composites

Appl. Phys. Lett. 88, 082907 (2006); 10.1063/1.2178582

MIT LINCOLN

LABORATORY CAREERS

Discover the satisfaction of innovation and service

to the nation
- Space Control

- Air \& Missile Defense

- Communications Systems \& Cyber Security

- Intelligence, Surveillance and

Reconnaissance Systems

- Advanced
Electronics
- Tactical Systems
- Homeland
Protection
- Air Traffic Control

LINCOLN LABORATORY

MassachusetTs Institute of TeChNOLOgY

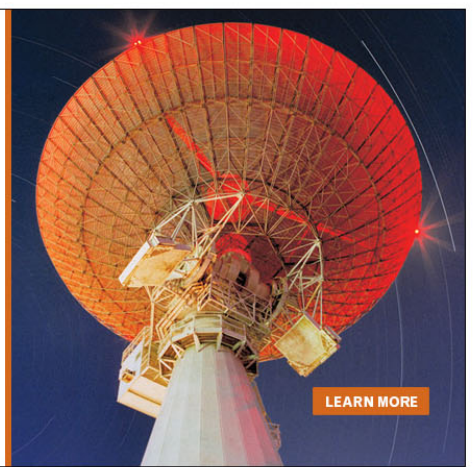




\title{
Near-flat self-biased magnetoelectric response in geometry gradient composite
}

\author{
Yuan Zhou a) and Shashank Priya a) \\ Bio-inspired Materials and Devices Laboratory (BMDL), Center for Energy Harvesting Materials and Systems \\ (CEHMS), Virginia Tech, Blacksburg, Virginia 24061, USA
}

(Received 5 February 2014; accepted 2 March 2014; published online 13 March 2014)

\begin{abstract}
We demonstrate a near-flat self-biased magnetoelectric (ME) effect in geometry gradient magnetostrictive-piezoelectric laminates. The near-flat behavior was characterized by a stable $\mathrm{ME}$ response over a wide range of magnetic DC bias. By adjusting the configuration of the magnetostrictive layer, we were able to control the magnitude of the self-biased magnetoelectric coefficient. The ME response was found to be almost independent of the applied DC bias in the range of $0 \sim 260$ Oe. This bandwidth was almost 650\% 3800\% higher than that of the conventional ME composites. This significant advancement opens great potential towards the development of high stability/sensitivity magnetic field sensors and energy harvesters. (C) 2014 AIP Publishing LLC. [http://dx.doi.org/10.1063/1.4868340]
\end{abstract}

\section{INTRODUCTION}

Magnetoelectric (ME) effect is the product property of ferromagnetic and piezoelectric materials that is expressed as polarization change in response to an externally applied magnetic field and vice-versa., ${ }^{1,2} \mathrm{ME}$ materials provide opportunity towards developing new applications such as tunable transformers, filters, resonators, and dual-phase energy harvesters. ${ }^{1-3}$ Prior research on ME materials has shown that bulk piezoelectric/magnetostrictive composites exhibit much stronger response than single-phase materials. $^{1,2}$ In the past decade, ME composites with various connectivity and structures have been developed in order to improve the ME coefficient and sensitivity. ${ }^{1,2,4}$ These experimental investigations have been complimented by the theoretical modeling which has led to the understanding of the role of various material and structural parameters. 5,6

In general, under a constant applied AC magnetic field, the ME coupling coefficient $\left(\alpha_{\mathrm{ME}}\right)$ shows a peak behavior in response to varying $\mathrm{DC}$ bias $\left(H_{\text {bias }}\right)$ and $\mathrm{AC}$ magnetic field frequency $(f)$. The frequency dependent behavior is related to the electromechanical resonance of the piezoelectric phase. The DC magnetic field dependence is due to the piezomagnetic coefficient variation as a function of $H_{\mathrm{dc}}$. The peak behavior presents a challenge as slight variations in the magnitude of the applied $H_{\text {bias }} / f$ could result in significant modulation in the magnitude of the $\alpha_{\mathrm{ME}}$. Thus, from the practical point of view two issues related to the conventional ME composite behavior should be resolved: (1) The requirement of $H_{\text {bias }}$ imposes limitation in the fabrication and miniaturization of the electronic components, and (2) the peak behavior limits the operating range around the optimal magnetic DC bias and AC frequency. These two issues should be addressed in order to open the application space for the ME composites.

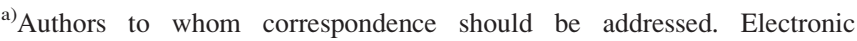
addresses: yzhou6@vt.edu and spriya@vt.edu
}

There have been few attempts in the literature towards addressing these two issues mainly focusing on the materials selection and composite architecture. ${ }^{1,7}$ These efforts have led to the reduction in the magnitude of the $H_{\text {bias }}$ from $6.8 \mathrm{kOe}$ to $5 \mathrm{Oe}$ by tuning the composition of the magnetostrictive phase and geometry of the ME composites. ${ }^{1,8}$ There has also been some progress in the design of self-biased ME composites that exhibit a large $\alpha_{\mathrm{ME}}$ near zero magnetic bias. ${ }^{9-11}$ For increasing the operating bandwidth of ME composites in terms of frequency, research has mainly focused on two concepts: one relies on merging the response of multiple laminates ${ }^{12}$ and the other on generating the multiple bending modes within the same laminate. ${ }^{13}$ However, prior efforts have not been successful in achieving a near flat ME response over a wide range of magnetic DC bias. In particular, self-biased ME effect with a near-flat DC bias response has never been reported to date.

In this paper, we report $\mathrm{ME}$ composites that exhibit flat and stable response over a wide range of magnetic DC bias. We further demonstrate the near-flat self-biased ME structure and describe the influence of the structural parameters on ME coefficient.

The question that needs to be answered is: "How to control the magnitude of optimum DC bias and ultimately create the self-biased magnetoelectric response?" Our recent study has revealed that by selecting $\mathrm{Ni}$ as the ferromagnetic phase, a self-biased magnetoelectric response can be obtained due to its non-zero piezomagnetic coefficient under the low field condition. ${ }^{11}$ Therefore, we used nickel as the magnetostrictive phase in the ME laminates. For adjusting the self-biased ME response, it was found that high magnetic flux concentration in the magnetic phase has positive effect. ${ }^{11,14}$ By considering the influence of demagnetization effect, the effective magnetic flux density can be expressed as ${ }^{11}$

$$
B_{\text {eff }}=\mu_{0}\left(H_{\text {eff }}+M\right)=\mu_{0}\left(H_{\text {bias }}+M\right)-\mu_{0} M N_{\mathrm{d}},
$$

where $H_{\text {eff }}$ is the internal effective magnetic field and can be written as 


$$
H_{\text {eff }}=H_{\text {bias }}-H_{\mathrm{d}}=H_{\text {bias }}-M N_{\mathrm{d}}
$$

$M$ is the magnetization, $H_{\text {bias }}$ is the applied external magnetic field, $H_{\mathrm{d}}$ is the demagnetization field proportional to the demagnetization factor $\left(N_{\mathrm{d}}\right)$. The demagnetization factor for a finite non-spherical ferromagnet was found to be dependent on its geometry. ${ }^{15}$ Thus, one can tune the magnitude of the self-biased ME response by modifying the $B$-field distribution through the variation in geometry of the ferromagnetic phase. In order to achieve the same magnitude of $H_{\text {eff }}$, one needs to apply larger $H_{\text {bias }}$ when $H_{\mathrm{d}} / N_{\mathrm{d}}$ is larger and vice-versa.

We estimated the magnetic flux density distribution of $\mathrm{Ni}$ by a magnetostatic simulation using the finite element model (FEM, ANSYS MAXWELL 15.0). Ni plate with different thickness $(t=0.2,0.4,0.6 \mathrm{~mm})$ but same planar dimension of $20 \times 5 \mathrm{~mm}^{2}$ and different length $(l=5,10$, $20 \mathrm{~mm}$ ) but same cross-section dimension of $5 \times 0.2 \mathrm{~mm}^{2}$ were used in this simulation. In this model, all Ni plates were placed in the air and assigned relative permeability of 600 and coercive field force (in the axial length direction) of $0.7 \mathrm{Oe}^{16}$ to approximate the real behavior of the Ni. A magnetostatic insulating boundary condition was applied to the $\mathrm{Ni}$. In each case, the nickel was meshed with a maximum element mesh size of $100 \mu \mathrm{m}$ and the simulations were completed within 1\% accuracy. Figures 1(a) and 1(b) show the simulated magnetic flux density distribution along Ni plates as function of thickness and length. We can clearly notice that thicker nickel exhibits weaker magnetic induction. The increase in length of the $\mathrm{Ni}$ resulted in enhancement in the magnitude of magnetic induction. These results led us to the assumption that by designing a composite with separate $\mathrm{Ni}$ sections in geometric gradient configuration, one can manipulate the self-biased $\mathrm{ME}$ response through combination of the output from separate sections of the composite.

\section{EXPERIMENTAL}

We designed and fabricated two different geometry gradient unimorph configurations [thickness gradient composite (TGC) and length gradient composite (LGC)] based on these simulations, as shown in Figs. 1(c) and 1(d). In Fig. 1(c), a Ni foil with thickness of $0.2 \mathrm{~mm}$ was first machined into a 王 structure with three beams of same lateral dimension $\left(20 \times 5 \mathrm{~mm}^{2}\right)$. Next, we stacked different number of Ni layers on each section to achieve a gradient thickness of $0.2,0.4$ and $0.6 \mathrm{~mm}$. A different configuration shown in Fig. 1(d) referred to as LGC, consists of a $0.2 \mathrm{~mm}$ thick $\mathrm{Ni}$ length gradient architecture with three beams of various lateral dimension $\left(5 \times 5,10 \times 5,20 \times 5 \mathrm{~mm}^{2}\right)$. All of the beams were separated by a bridge of dimension $5 \times 5 \times 0.2 \mathrm{~mm}^{3}$. The contour plots illustrate the magnitude of magnetic flux density distribution along the gradient structure. Transversely poled lead zirconate titanate (PZT) based piezoelectric plates (PSI-5A4E, Piezo System) with dimension of $10 \times 5 \times 0.2 \mathrm{~mm}^{3}$ were attached in the center of each beam using epoxy (West System, USA, with curing at room temperature for $24 \mathrm{~h}$ ). In both TGC and LGC, the piezoelectric plates were electrically connected in parallel. The ME voltage coefficient was measured by applying the DC magnetic field $\left(H_{\mathrm{dc}}\right)$ longitudinally with the sample placed in the center of the Helmholtz coil under an AC magnetic field $\left(H_{\mathrm{ac}}\right)$. ME voltage induced on the laminate was monitored using lock-in amplifier, and the strength of the $H_{\mathrm{dc}}$ was monitored using Gauss meter (F. W. BELL, Model 6010).

\section{RESULTS AND DISCUSSION}

We first characterized the ME voltage coefficient of the thickness and length gradient composites as function of $H_{\mathrm{dc}}$ with $H_{\mathrm{ac}}=1 \mathrm{Oe}$ at $1 \mathrm{kHz}$, as shown in Figs. 2(a) and 2(b). For comparison, the ME coefficient from each Ni/PZT section was also measured separately. In all cases, the magnitude of $\alpha_{\mathrm{ME}}$ shows hysteretic behavior during $H_{\mathrm{dc}}$ sweep, in
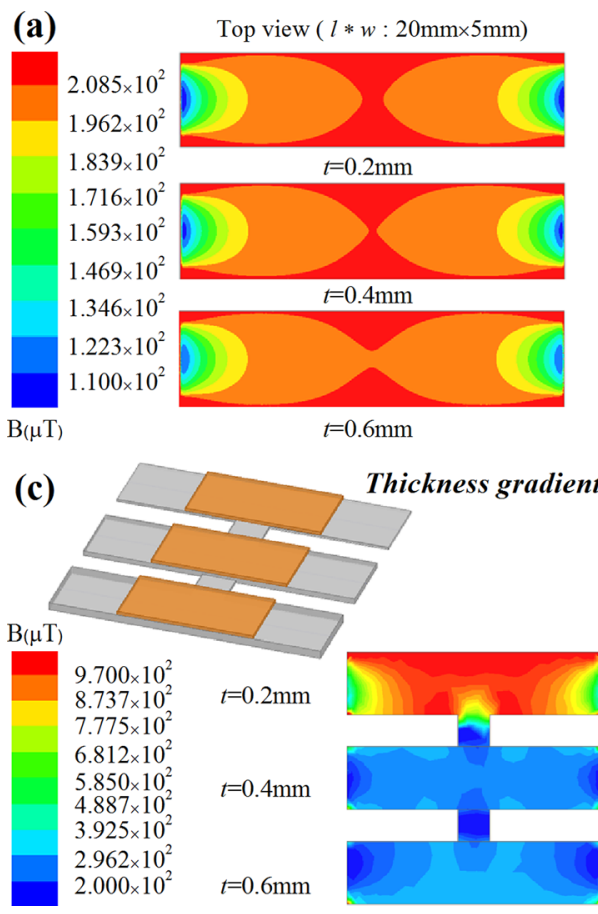

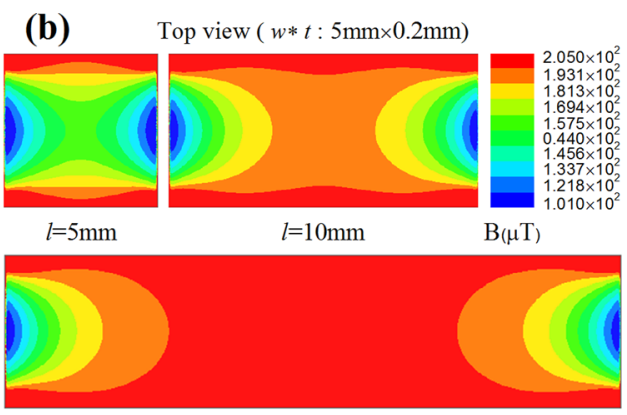

$l=20 \mathrm{~mm}$

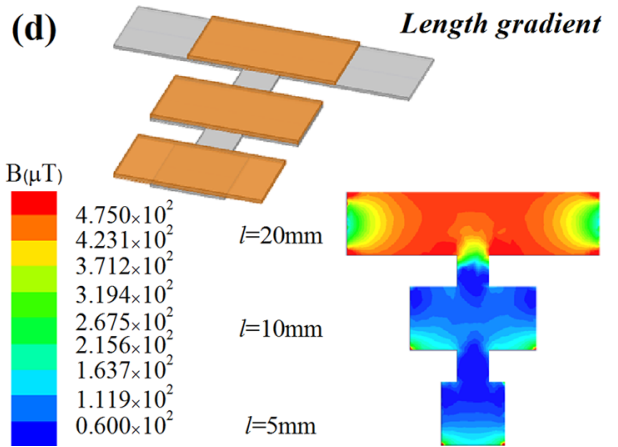

FIG. 1. FEM simulation data for inplane magnetic flux density distribution along the Ni plates with (a) variation in thickness, and (b) variation in length. Schematic diagram of the geometry gradient magnetoelectric laminates depicting (c) a thickness gradient composite with three beams of different thickness, and (d) a length gradient composite with three beams of different length. The inset contour plots show the corresponding simulated magnetic field strength distribution using ANSYS MAXWELL. 

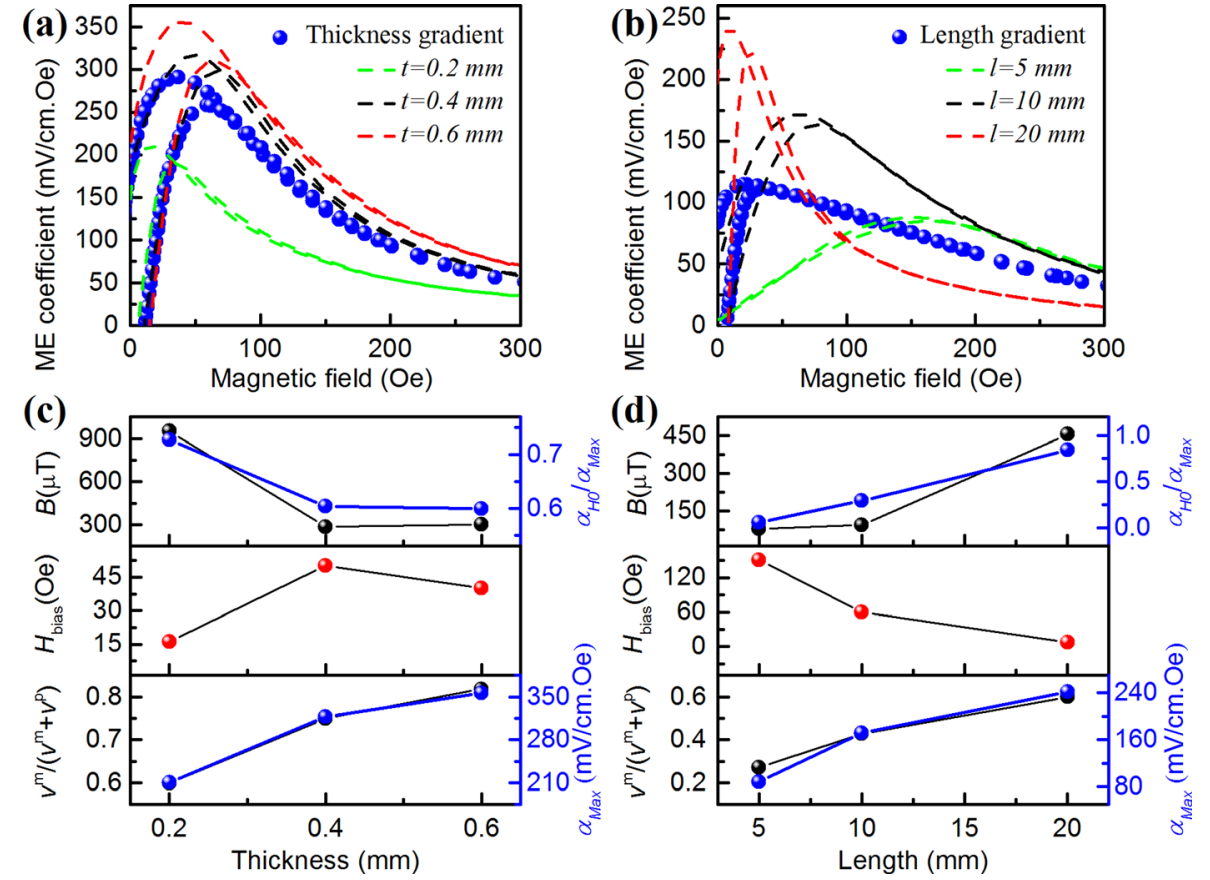

FIG. 2. ME voltage coefficient of the geometry gradient composites: (a) thickness gradient composite and its corresponding ME behavior with varying thickness, and (b) length gradient composite and its corresponding ME behavior with varying length. Characteristic ME response from each Ni/PZT section of the geometry gradient composites (c) as a function of Ni thickness, and (d) as a function of Ni length. All the measurements were taken under $H_{\mathrm{ac}}=1 \mathrm{Oe}$ at $1 \mathrm{kHz}$. agreement with our prior study on Ni-piezoelectric laminates. ${ }^{11}$ Large self-biased ME response $\left(\alpha_{\mathrm{HO}}\right)$ was obtained with the magnitude of $\sim 183 \mathrm{mV} \mathrm{cm}^{-1} \mathrm{Oe}^{-1}$ (TGC) and $\sim 86 \mathrm{mV} \mathrm{cm}^{-1} \mathrm{Oe}^{-1}$ (LGC), respectively. The ME bandwidth $(\Delta H)$ of the composite is defined as the DC magnetic bias difference between the lower and upper half power points $(3 \mathrm{~dB})$ where the ME coefficient has dropped by a factor of $1 / \sqrt{2}$ or 0.707. Benefit from the self-biased hysteretic behavior, both for TGC $(\Delta H=97.5 \mathrm{Oe})$ and LGC $(\Delta H=137.3 \mathrm{Oe})$, is illustrated in terms of the larger bandwidth than the conventional ME composite $(\Delta H=6.9 \mathrm{Oe}){ }^{8}{ }^{8}$ It should be noted that TGC and LGC exhibited reduced ME coefficient in comparison to the response from the individual sections in the composite due to the charge redistribution occurring from the parallel electrical connection.

Figs. 2(c) and 2(d) summarize the individual ME responses from separate sections of the composite. From these results, several important observations can be made: (1) Thinner or longer Ni plate possesses larger magnetic flux density, (2) laminates with stronger $B$-field exhibit notably higher self-biased ME coefficient $\left(\alpha_{\mathrm{Ho}} / \alpha_{\mathrm{Max}}\right)$ while requiring lower $H_{\text {bias }}$, (3) the magnitude of $\alpha_{\text {Max }}$ increases dramatically as the magnetic phase volume ratio increases, (4) magnitude of individual response from separate sections of the composite plays an important role towards determining the total ME response.
Guided by these results, we redesigned the dimensional gradient composite having a larger geometry separation to generate a broadband response. In this new design referred to as complex gradient composite (CGC), one short and thick beam $\left(10 \times 5 \times 0.6 \mathrm{~mm}^{3}\right)$ was connected with a long and thin beam $\left(20 \times 5 \times 0.2 \mathrm{~mm}^{3}\right)$ as shown in Fig. 3(a). Large variation of magnetic flux density was realized in each section of the CGC. The ME coefficient of the composite demonstrated a large self-biased effect $\left(a_{\mathrm{H} 0} / a_{\mathrm{Max}}=0.72\right)$, as shown in Fig. 3(b). The ME response of CGC is combined behavior of the two sections. The individual peaks corresponding to each section were brought in vicinity of each other by modulating the geometry resulting in a flat response as function of $H_{\mathrm{dc}}$. This result clearly demonstrates the wideband self-biased magnetoelectric response in CGCs.

Next, we investigated the effect of AC magnetic field frequency and DC magnetic field bias on the ME response. We first show the ME coefficient of each section in the CGC [Fig. 4(a)]. The first peak $\left(139 \mathrm{mV} \mathrm{cm}^{-1} \mathrm{Oe}^{-1}\right.$ at $\left.H_{\text {bias }}=30 \mathrm{Oe}\right)$ and the second peak $\left(120 \mathrm{mV} \mathrm{cm}^{-1} \mathrm{Oe}^{-1}\right.$ at $\left.H_{\text {bias }}=159 \mathrm{Oe}\right)$ were associated with the large and small beam component of the CGC respectively. The combined response of the structure exhibited a large bandwidth of $\sim 260$ Oe which is almost 38 times of the conventional Metglas/PZT composite. ${ }^{8}$ Next, we measured the ME (a)

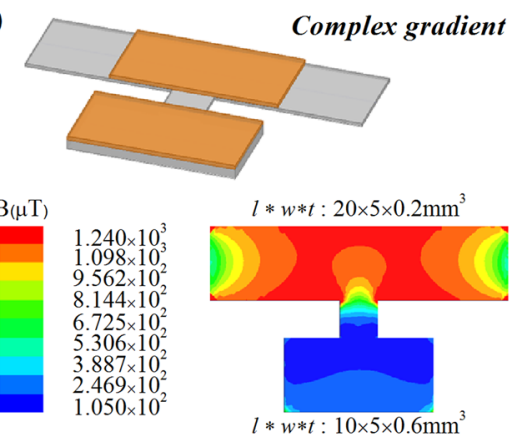

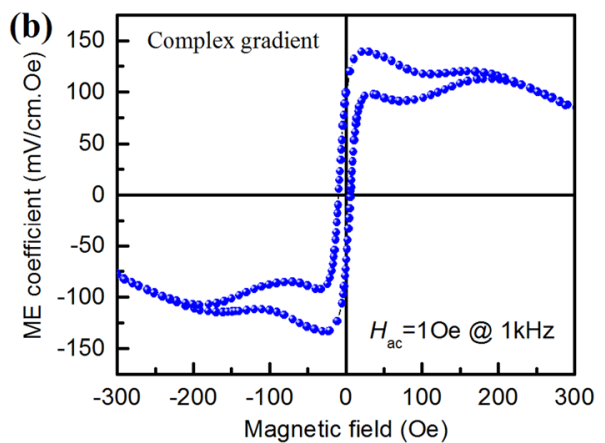

FIG. 3. (a) Schematic diagram and the simulated magnetic flux density distribution of the complex gradient magnetoelectric composite, (b) ME voltage coefficient of the complex gradient composite as a function of DC magnetic field under $H_{\mathrm{ac}}=1 \mathrm{Oe}$ at $1 \mathrm{kHz}$. 


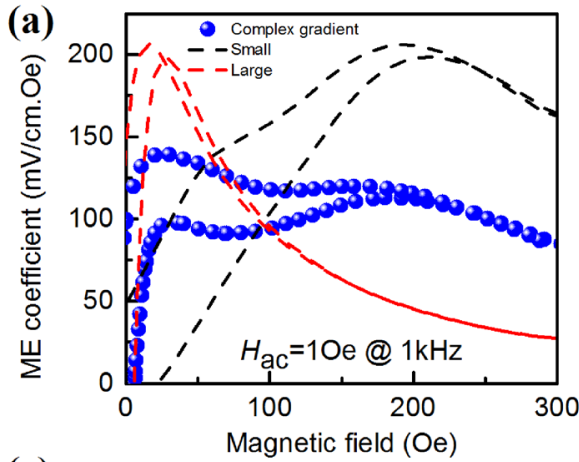

(c)

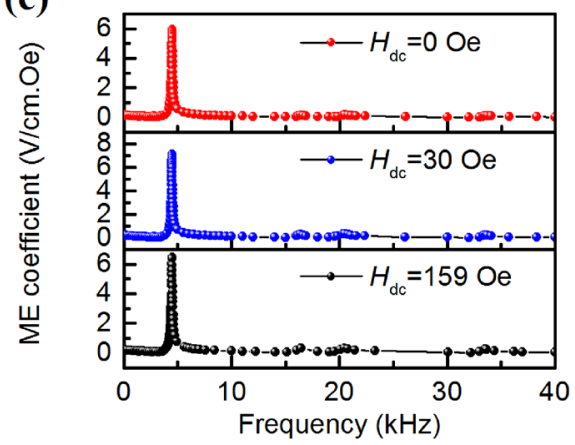

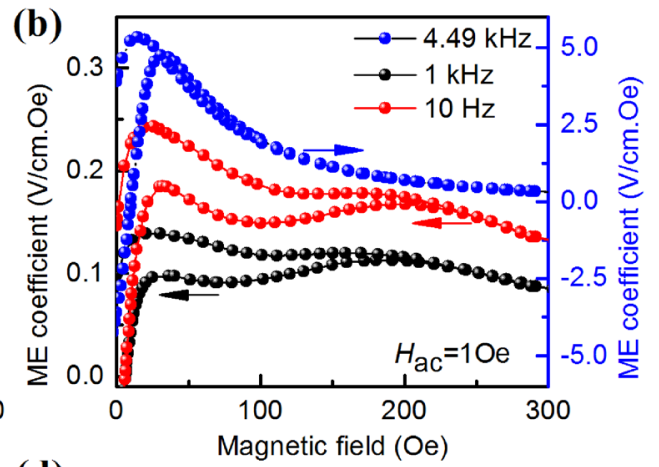

(d)

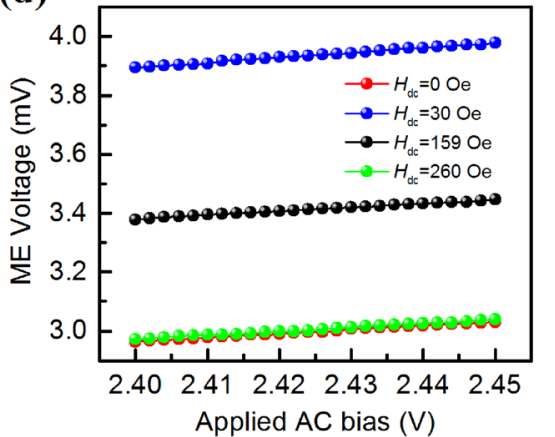

FIG. 4. ME response of the complex gradient composite: (a) ME voltage coefficient of the CGC and its corresponding individual section ME behavior with varying $\mathrm{DC}$ magnetic field under $H_{\mathrm{ac}}=1 \mathrm{Oe}$ at $1 \mathrm{kHz}$, (b) $\mathrm{ME}$ voltage coefficient at low frequency $(10 \mathrm{~Hz})$, off-resonance frequency $(1 \mathrm{kHz})$ and resonance frequency $(4.49 \mathrm{kHz})$ as a function of DC magnetic field with $H_{\mathrm{ac}}=1 \mathrm{Oe}$, (c) ME voltage coefficient as a function of applied AC frequency with $H_{\mathrm{dc}}=0$, 30 , and $159 \mathrm{Oe}$, respectively, and (d) ME voltage output measured at $H_{\mathrm{dc}}=0,30,159$, and $260 \mathrm{Oe}$ under various applied AC voltage on Helmholtz coil $\left(H_{\mathrm{ac}}\right)$. coefficient at low frequency $(10 \mathrm{~Hz})$, off-resonance frequency $(1 \mathrm{kHz})$ and at the resonance frequency $(4.49 \mathrm{kHz})$ as a function of $H_{\mathrm{dc}}$, as shown in Fig. 4(b). The near-flat ME behavior was found to occur at all the frequencies with enhanced ME coefficient. The $\alpha_{\text {Max }}$ was improved exhibiting the magnitude of $0.24 \mathrm{~V} \mathrm{~cm}^{-1} \mathrm{Oe}^{-1}$ and $5.35 \mathrm{~V} \mathrm{~cm}^{-1} \mathrm{Oe}^{-1}$ at $10 \mathrm{~Hz}$ and $4.49 \mathrm{kHz}$. To investigate the stability of self-biased ME response, we measured the ME coefficient as a function of frequency under various $H_{\mathrm{dc}}=0,30,159 \mathrm{Oe}$, as shown in Fig. 4(c). All the ME responses followed the similar trend and had similar range of $\alpha_{\mathrm{ME}}\left(\sim 6 \mathrm{~V} \mathrm{~cm}^{-1} \mathrm{Oe}^{-1}\right)$ at $\sim 4.49 \mathrm{kHz}$, even without external $H_{\mathrm{dc}}$. The excellent stability of the self-biased ME response in this design offers significant advantage towards developing magnetoelectric energy harvester ${ }^{3,17}$ that can scavenge the magnetic field energy regardless of the ambient $H_{\mathrm{dc}}$. It should be noted that prior research on the magnetoelectric harvesters has revealed that the requirement of $H_{\text {bias }}$ is problematic towards high power density. ${ }^{18,19}$ The structure can also be used to detect the external $H_{\mathrm{ac}}$ by monitoring the variation of ME voltage based on the conversion ratio between the two parameters, $\alpha_{\mathrm{ME}}=\delta E_{\mathrm{ac}} / \delta H_{\mathrm{ac}}$. In Fig. $4(\mathrm{~d})$, we measured the ME voltage output as function of applied $H_{\mathrm{ac}}$ under wide range of $H_{\mathrm{dc}}$ from 0 Oe to 260 Oe. Linear increase in ME voltage output was observed under various $H_{\mathrm{dc}}$ with similar slope as the applied AC voltage was varied in the Helmholtz coil, indicating a small variation of $\alpha_{\mathrm{ME}}$ regardless of the magnitude of external $H_{\mathrm{dc}}$. This result suggests a great potential towards implementing this structure into a high stability/sensitivity AC magnetic field sensor.

Table I lists the ME properties of various ME composites, including conventional Metglas/PZT laminates $(\mathrm{c}-\mathrm{ME}),{ }^{8,11}$ wideband ME laminate (w-ME), ${ }^{7}$ co-fired ME composite (Co-fired) ${ }^{20}$ self-biased ME composite (s-ME), ${ }^{11}$ and geometry gradient ME composites (TGC, LGC, and CGC). The effective DC bias response stability quality factor of the ME laminate can be expressed as $Q=\Delta H / H_{\text {bias }}$, where large $Q$ factor with low $H_{\text {bias }}$ and high $\Delta H$ is desired. Following conclusion can be drawn from this table: (1) Compared to conventional $\mathrm{ME}$ composites, giant $\Delta H$ with the magnitude of 261.3 Oe was achieved in complex gradient composite, which is $\sim 650 \%-3800 \%$ higher, (2) all the self-biased ME composites exhibited a higher magnitude of $Q$ compared to conventional ME composites, indicating either a much wider bandwidth or a lower $H_{\text {bias }}$, (3) geometry gradient structure dramatically enhances the DC bias

TABLE I. Magnetoelectric properties of various ME composites.

\begin{tabular}{lccccc}
\hline \hline Sample & $H_{\text {bias }}[\mathrm{Oe}]$ & $\Delta H[\mathrm{Oe}]$ & $Q=\Delta H / H_{\text {bias }}$ & $a_{H 0}[\mathrm{mV} / \mathrm{cm} \mathrm{Oe}]$ & $a_{\text {Max }}[\mathrm{mV} / \mathrm{cm} \mathrm{Oe}]$ \\
\hline c-ME $^{8}$ & 5 & 6.9 & 1.4 & $\sim 0$ & $22 \times 10^{3}$ \\
c-ME $^{11}$ & 69.0 & 39.7 & 0.6 & $\sim 0$ & 154.66 \\
w-ME $^{7}$ & 220 & $\sim 122$ & 0.6 & $\sim 0$ & 88 \\
Co-fired $^{7}$ & 3.0 & 33.9 & 11.3 & 1316.4 & 1347.1 \\
s-ME $^{11}$ & 15.5 & 89.0 & 2.6 & 1254.0 & 1381.2 \\
TGC & 37.0 & 137.3 & 6.1 & 183.4 & 290.7 \\
LGC & 22.4 & 261.3 & 8.7 & 96.0 & 114.6 \\
CGC & 30.1 & & & 99.4 & 0.98 \\
\hline \hline
\end{tabular}


response stability of ME composite. Thus, the combination of self-biased ME effect and geometry gradient structure not only helps in eliminating the need for DC magnetic bias, but more importantly enhances the ME response stability in a wide range of DC bias.

\section{SUMMARY}

In summary, we designed and fabricated a series of geometry gradient Ni/PZT composites. The design was able to provide a near-flat self-biased ME response. By optimizing the structural and geometrical parameters of the ferromagnetic phase, a significantly flat and stable self-biased ME response over a wide DC magnetic bias $(\Delta H=261.3 \mathrm{Oe})$ was demonstrated. This near-flat self-biased ME behavior provides great advancement towards development of the high stability/sensitivity magnetoelectric energy harvester and $\mathrm{AC}$ magnetic field sensor.

\section{ACKNOWLEDGMENTS}

The authors gratefully acknowledge the financial support from the Office of Basic Energy Science, Department of Energy (DE-FG02-06ER46290) and the NSF INAMM program (S.P.). The authors also thank the Office of Naval Research for supporting the research (Y. Zhou) through Center for Energy Harvesting Materials and Systems.

${ }^{1}$ C. W. Nan, M. I. Bichurin, S. X. Dong, D. Viehland, and G. Srinivasan, J. Appl. Phys. 103, 031101 (2008).
${ }^{2}$ S. Priya, R. Islam, S. X. Dong, and D. Viehland, J. Electroceram. 19, 149 (2007).

${ }^{3}$ Y. Zhou, D. J. Apo, and S. Priya, Appl. Phys. Lett. 103, 192909 (2013).

${ }^{4}$ G. Srinivasan, Annu. Rev. Mater. Res. 40, 153 (2010).

${ }^{5}$ G. Srinivasan, E. T. Rasmussen, B. J. Levin, and R. Hayes, Phys. Rev. B 65, 134402 (2002).

${ }^{6}$ M. I. Bichurin, D. A. Filippov, V. M. Petrov, V. M. Laletsin, N. Paddubnaya, and G. Srinivasan, Phys. Rev. B. 68, 132408 (2003).

${ }^{7}$ C. S. Park, C. W. Ahn, J. Ryu, W. H. Yoon, D. S. Park, H. E. Kim, and S. Priya, J. Appl. Phys. 105, 094111 (2009).

${ }^{8}$ S. X. Dong, J. Y. Zhai, J. F. Li, and D. Viehland, Appl. Phys. Lett. 89, 252904 (2006).

${ }^{9}$ S. K. Mandal, G. Sreenivasulu, V. M. Petrov, and G. Srinivasan, Appl. Phys. Lett. 96, 192502 (2010).

${ }^{10}$ S. C. Yang, C. S. Park, K. H. Cho, and S. Priya, J. Appl. Phys. 108, 093706 (2010).

${ }^{11}$ Y. Zhou, S. C. Yang, D. J. Apo, D. Maurya, and S. Priya, Appl. Phys. Lett. 101, 232905 (2012).

${ }^{12}$ H. Yu, M. Zeng, Y. Wang, J. G. Wan, and J. M. Liu, Appl. Phys. Lett. 86, 032508 (2005).

${ }^{13}$ D. R. Patil, R. C. Kambale, Y. Chai, W. H. Yoon, D. Y. Jeong, D. S. Park, J. W. Kim, J. J. Choi, C. W. Ahn, B. D. Hahn, S. J. Zhang, K. H. Kim, and J. Ryu, Appl. Phys. Lett. 103, 052907 (2013).

${ }^{14}$ Z. Fang, S. G. Lu, F. Li, S. Datta, Q. M. Zhang, and M. El Tahchi, Appl. Phys. Lett. 95, 112903 (2009).

${ }^{15}$ C. M. Chang and G. P. Carman, Phys. Rev. B 76, 134116 (2007).

${ }^{16}$ W. F. Brown, Handbook of Chemistry and Physics, edited by E. U. Condon and H. Odishaw (McGraw-Hill, New York, 1958), Ch. 8.

${ }^{17}$ Y. Zhou, A. Bhalla, and S. Priya, Mater. Res. Soc. Symp. Proc. 1556, mrss13-1556-w01-03 (2013).

${ }^{18}$ S. X. Dong, J. Y. Zhai, J. F. Li, D. Viehland, and S. Priya, Appl. Phys. Lett. 93, 103511 (2008).

${ }^{19}$ R. C. Kambale, W. H. Yoon, D. S. Park, J. J. Choi, C. W. Ahn, J. W. Kim, B. D. Hahn, D. Y. Jeong, B. C. Lee, G. S. Chung, and J. Ryu, J. Appl. Phys. 113, 204108 (2013).

${ }^{20}$ Y. K. Yan, Y. Zhou, and S. Priya, Appl. Phys. Lett. 102, 052907 (2013). 